\title{
Cancer Antigen 15-3 Serum Level as a Biomarker for Advanced Micropapillary Urothelial Carcinoma of the Bladder: A Case Report
}

\author{
Koichiro Takayama ${ }^{a}$ Shintaro Narita ${ }^{b}$ Yasushiro Teraic ${ }^{c}$ Ryoko Saito $^{d}$ \\ Tomonori Habuchi ${ }^{b}$ \\ aDepartment of Urology, Yokote Municipal Hospital, Akita, Japan; b Department of Urology, \\ Akita University School of Medicine, Akita, Japan; 'Department of Urology, Towada City \\ Hospital, Aomori, Japan; dDepartment of Pathology, Tohoku University School of Medicine, \\ Miyagi, Japan
}

\author{
Keywords \\ Micropapillary urothelial carcinoma $\cdot$ Bladder cancer $\cdot$ CA 15-3 $\cdot$ MUC1
}

\begin{abstract}
A 73-year-old woman with no history of disease was referred to our hospital with fatigue and joint pain. Screening blood test showed that her cancer antigen 15-3 (CA 15-3) serum level was elevated to $36.6 \mathrm{U} / \mathrm{mL}$, and a contrast-enhanced computed tomography scan revealed a bladder tumor without metastasis. Cystoscopy showed a papillary and a small kissing tumor, and the histopathological analysis of the bladder tumor obtained by transurethral resection (TUR) showed invasive urothelial carcinoma (UC) with micropapillary variant (pT1). At 4 weeks after TUR, the CA 15-3 serum level was markedly increased to $180.6 \mathrm{U} / \mathrm{mL}$, and radiographic examinations revealed multiple regional and nonregional lymph node metastases. The patient received systemic therapy with gemcitabine and cisplatin. After 3 cycles of chemotherapy, the size of all lymph node metastases reduced by $80 \%$ in diameter, and the CA $15-3$ serum level decreased from 238.2 to $11.4 \mathrm{U} / \mathrm{mL}$. Immunohistological analysis showed that the bladder tumor was positive for mucin 1, of which CA 15-3 is an epitope. In our patient, changes in the CA 15-3 serum levels were in congruence with the clinical course of advanced micropapillary UC (MPUC). Therefore, the CA 15-3 serum level may be a potentially valuable biomarker for MPUC.
\end{abstract}




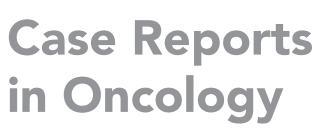

\section{Introduction}

Micropapillary urothelial carcinoma (MPUC) of the bladder is a rare variant, accounting for $0.7-8.3 \%$ of all urothelial carcinomas (UCs) $[1,2]$. Patients with MPUC have a higher rate of muscle invasion, lymph node metastasis, and distal metastasis compared with patients with conventional UC [2]. Thus, MPUC is usually diagnosed at an advanced stage and follows an aggressive clinical course. Pathologically, MPUC is characterized by the lack of true fibrovascular cores and the formation of small clusters of high-grade tumor cells contained within the lacunar spaces [1]. Given that MPUC has a poor prognosis compared with that of conventional UC [2], useful biomarkers and optimal treatments for MPUC are urgently needed. Here, we report a patient with advanced MPUC whose cancer antigen 15-3 (CA 15-3) serum levels showed a substantial congruence with the disease status.

\section{Case Report}

A 73-year-old woman was referred to our hospital with fatigue and joint pain. Until the symptoms appeared, the patient had a normal health status and no history of cancer. Serum tumor markers were measured to screen for malignant disease. The screening test results that her CA 15-3 serum level was elevated to $36.6 \mathrm{U} / \mathrm{mL}$ (Reference range: $<31.3 \mathrm{U} / \mathrm{mL}$ ). A contrast-enhanced computed tomography (CT) scan showed a bladder tumor measuring 16 $\mathrm{mm}$ in diameter with no metastases (Fig. 1a). Cystoscopy revealed a papillary and a small kissing tumor in the bladder neck (Fig. 1b). The patient underwent transurethral resection (TUR) of the bladder. The histopathological diagnosis of the resected specimen was invasive UC with micropapillary variant (pT1) according to the presence of tumor cell clusters within clear spaces shown by hematoxylin-eosin staining (Fig. 2a). An immunohistochemical analysis using mucin 1 (MUC1) antibody (Ma695; Novocastra Laboratories Ltd., Newcastle upon Tyne, UK) showed that MUC1, which has been observed in invasive micropapillary carcinoma [3], was expressed in the cell membrane of the tumor cell clusters (Fig. 2b). Although we considered the disease organ-confined, a fluorodeoxyglucose positron emission tomography with CT scan performed 4 weeks after TUR showed multiple regional pelvic metastases in addition to nonregional para-aortic lymph node metastases (Fig. 3a-d). At that time, the CA 15-3 serum level was markedly elevated to $180.6 \mathrm{U} / \mathrm{mL}$. Consequently, we diagnosed her condition as cT2N1M1 urothelial bladder cancer with micropapillary variant. Systemic chemotherapy consisting of gemcitabine $\left(1,000 \mathrm{mg} / \mathrm{m}^{2}\right.$ on days 1,8 , and 15$)$ and cisplatin $\left(70 \mathrm{mg} / \mathrm{m}^{2}\right.$ on day 2) was administered. After 3 cycles of chemotherapy, a CT scan showed that the diameter of the lymph node masses had shrunk by $80 \%$ (Fig. 3e, f). At this time, the CA 15-3 serum level had decreased from 238.2 to $11.4 \mathrm{U} / \mathrm{mL}$ in congruence with tumor shrinkage (Fig. 3g). The patient declined further treatment and received best supportive care at another clinic.

\section{Discussion/Conclusion}

CA 15-3 is a common tumor marker in advanced breast cancer [4]. Elevated CA 15-3 levels were reported in other malignancies such as ovarian, lung, and liver cancers [5]. To our knowledge, this is the first report to demonstrate that changes in CA 15-3 serum levels reflected the clinical course in a patient with advanced MPUC, suggesting that CA 15-3 serum level may be a potential biomarker of MPUC.

MUC1 is a glycoprotein expressed on the apical surface of normal epithelial cells and is involved in the maintenance of lumen formation [6]. CA 15-3 serum levels indicate a distinct

\section{Karger'}




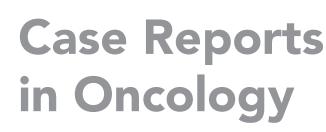

\begin{tabular}{l|l}
\hline Case Rep Oncol 2021;14:1019-1024 \\
\hline DOI: 10.1159/000515781 & $\begin{array}{l}\text { @ 2021 The Author(s). Published by S. Karger AG, Basel } \\
\text { www.karger.com/cro }\end{array}$ \\
\hline
\end{tabular}

Takayama et al.: Cancer Antigen 15-3 as a Biomarker for Micropapillary Urothelial Carcinoma
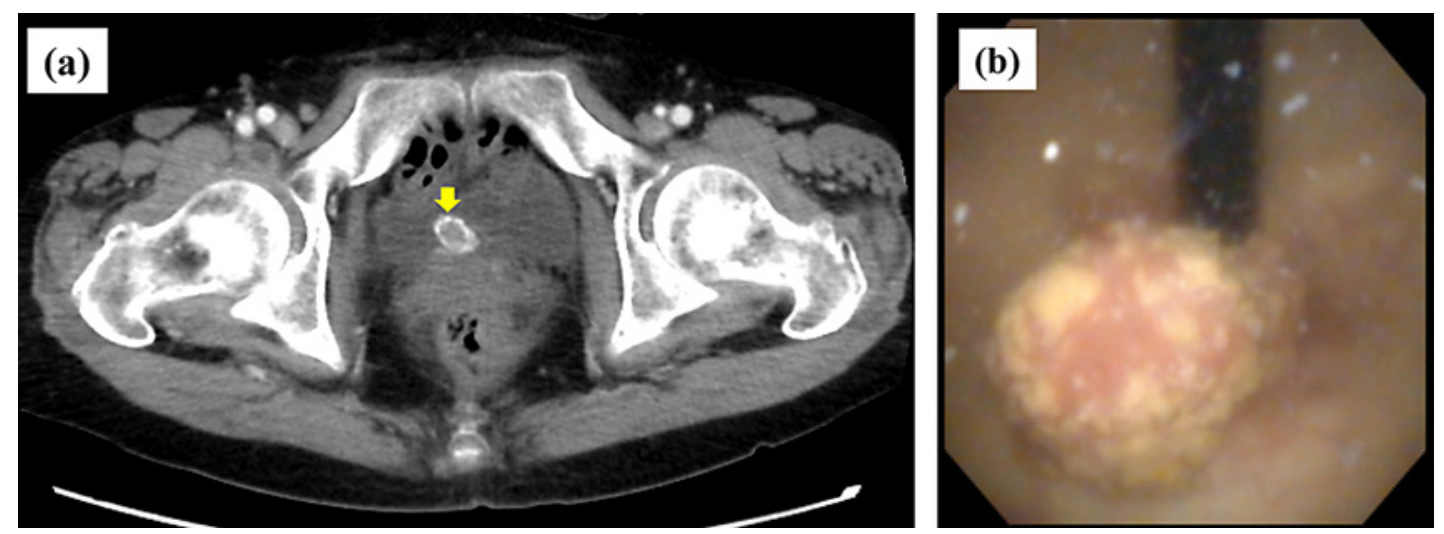

Fig. 1. CT and cystoscopy findings at the patient's first visit. a CT showed a bladder tumor with no metastases. The arrow indicates the bladder tumor. $\mathbf{b}$ Cystoscopy revealed a papillary tumor with calcification at the bladder neck. CT, computed tomography.
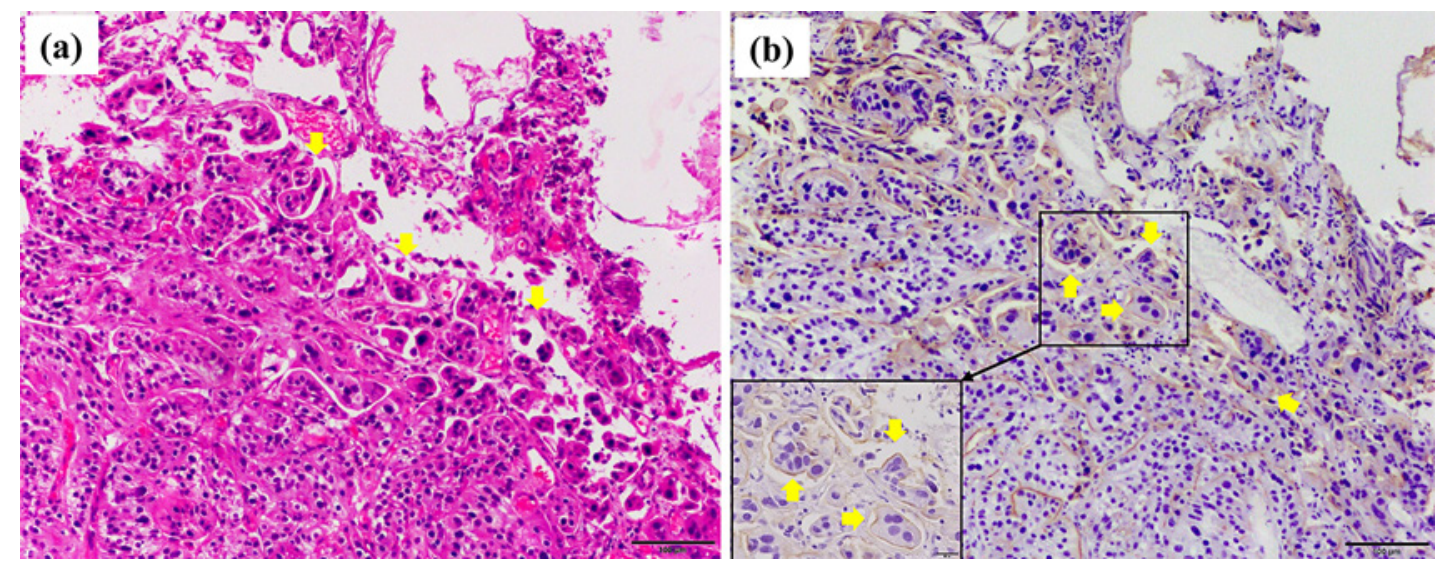

Fig. 2. Histopathological findings. a The arrows indicate clusters of micropapillary tumor cells nesting within clear spaces in the stroma in hematoxylin-eosin staining. $\mathbf{b}$ The arrows indicate that mucin 1 is expressed in the cell membranes of the micropapillary tumor cell clusters.

epitope on MUC1 and are used to determine MUC1 serum levels by immunoassay using monoclonal antibodies [4]. Upregulation and abnormal glycosylation of MUC1 were reported in several common cancers including breast, lung, colon, ovary, and bladder cancer [7]. Simms et al. [7] used a C595-based enzyme-linked immunosorbent assay to investigate MUC1 serum levels as a potential biomarker for transitional cell carcinoma of the bladder. When the MUC1 serum levels in 87 patients with transitional cell carcinoma of the bladder were compared with those of 31 controls with benign conditions, the overall sensitivity was only $24 \%$ for all tumors. The study concluded that the MUC1 serum level was not a useful tumor marker for screening but that MUC1 serum levels were high in T4 disease. Taking the results of our case into account, MUC1 and CA 15-3 serum levels may be useful for monitoring advanced disease, though not for screening of MPUC.

Immunohistochemical analysis of lung adenocarcinoma showed that MUC1 was strongly expressed across the surface of the micropapillary structure [8]. In contrast, there is less evidence regarding the impact of MUC1 expression in MPUC [9]. Sangoi et al. [9] proposed that MUC1 expression by immunohistochemistry could be used to discriminate invasive UC from artifact. Their study showed that MUC1 expression was significantly higher in invasive 

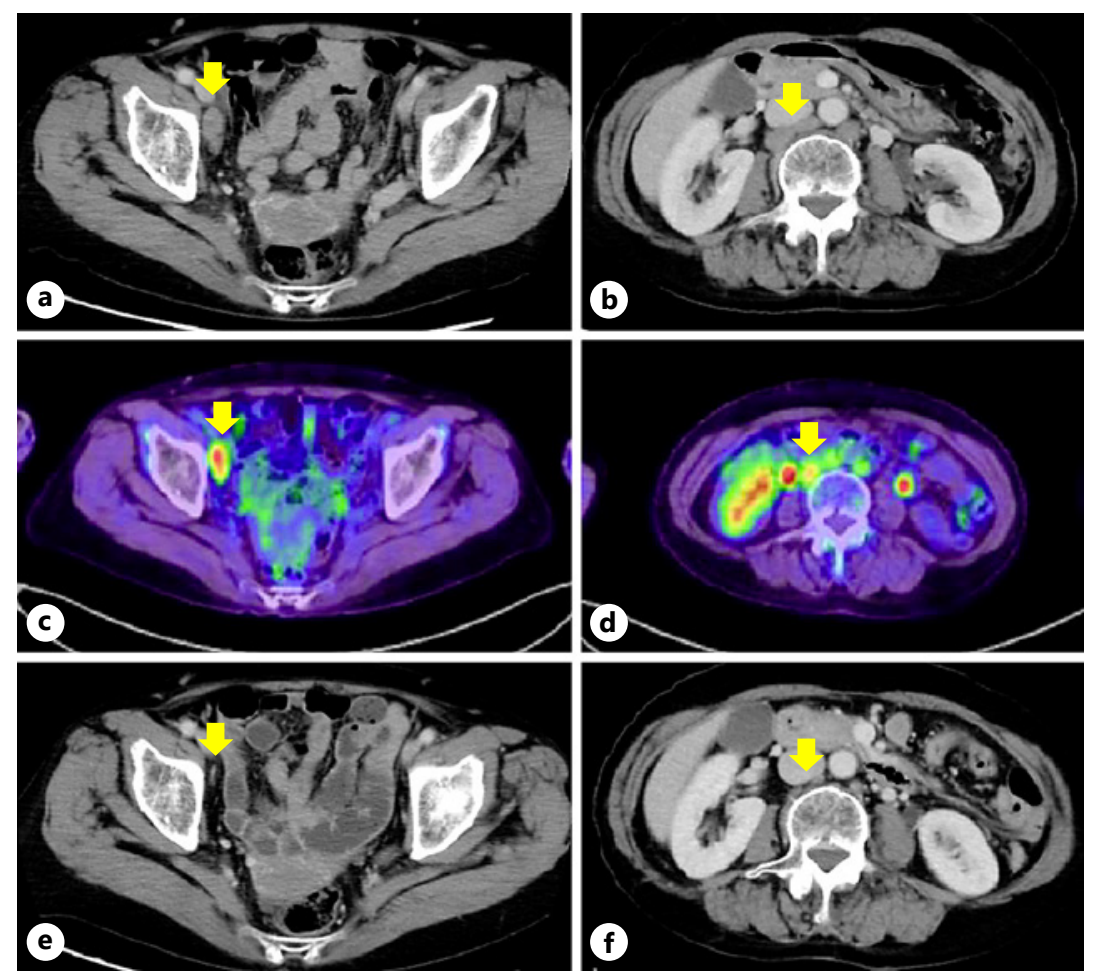

\section{CA15-3 (U/mL)}

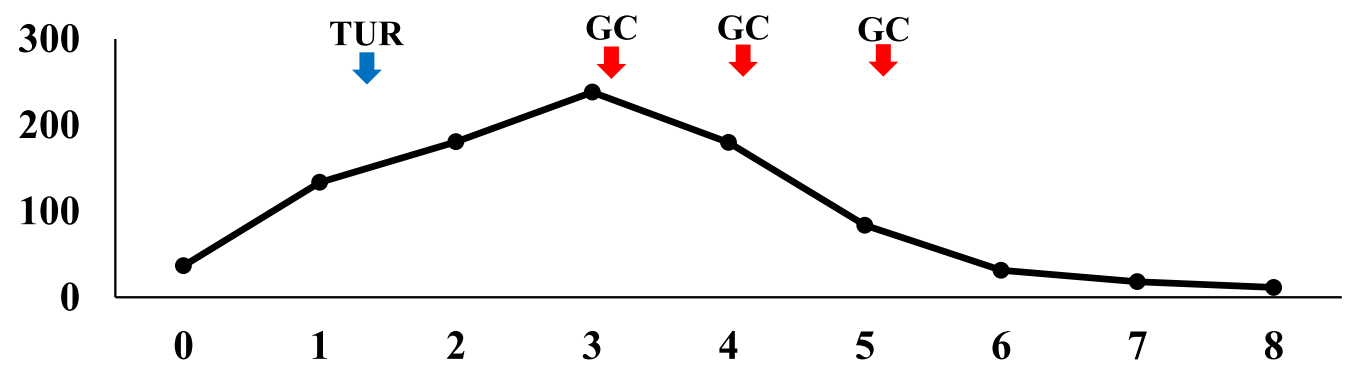

g

Time after initial visit (months)

Fig. 3. CT showed multiple regional (a) and nonregional (b) lymph node metastases before chemotherapy. Fluorodeoxyglucose positron emission tomography with CT showed multiple regional (c) and nonregional (d) lymph node metastases before chemotherapy. The lymph node metastases shrank after 3 cycles of chemotherapy (e, f). The arrows indicate the lymph node metastases. $\mathbf{g}$ Changes in CA 15-3 serum levels during the clinical course. TUR, transurethral resection; GC, combination chemotherapy with gemcitabine and cisplatin; CA 15-3, cancer antigen 15-3; CT, computed tomography.

micropapillary carcinoma than that in typical invasive UC ( $p=0.0102)$; however, the specificity was rather low (37\%). Consistent with the previous study, high MUC1 expression in the bladder tumor was seen in our patient with aggressive MPUC. Assessing the mechanical and clinical roles of MUC1 in MPUC in future studies will be intriguing. Furthermore, further analyses are needed to assess the correlation between tumor MUC1 expression and CA 15-3 serum levels in patients with advanced MPUC. Recently, several studies have proposed 
potential biomarkers for MPUC such as human epidermal growth factor receptor-2 overexpression [10] and $m i R-296$ and RUVBL1 activation [11]. Although these candidates are potentially useful for screening and follow-up in patients with MPUC in the future, they are currently not easy to measure in clinical practice. On the other hand, CA 15-3 is one of the most widely used tumor markers in clinical practice [4]. Therefore, the current results may provide physicians with a tool for more precise posttreatment intervention in patients with MPUC.

The MPUC in our patient responded successfully to systemic chemotherapy with gemcitabine and cisplatin. The optimal treatment strategy for MPUC remains controversial because most of the previous studies have targeted localized diseases [12]. Neoadjuvant chemotherapy using a platinum-based regimen induced complete pathological response in MPUC although whether there is a direct survival benefit remains unknown [13]. In the largest single-center study, a complete pathological response was observed in $52 \%$ of patient with micropapillary tumors who received neoadjuvant chemotherapy compared with $19 \%$ of patients with micropapillary tumors who had immediate cystectomy without neoadjuvant chemotherapy after TUR [14]. Recently, Deuker et al. [15] conducted an observational study of 120,491 patients with bladder cancer who were included in the surveillance epidemiology and results registry. The study demonstrated that in the metastatic stage, chemotherapy significantly improved cancer-specific mortality-free survival (hazard ratio $0.36, p=0.04$ ) in patients with MPUC compared with that in patients with conventional UC. Furthermore, longer median cancer-specific mortality-free survival was associated with radical cystectomy combined with chemotherapy than radical cystectomy alone in patients with nonorganconfined MPUC (hazard ratio 0.69, $p=0.2$ ). These results and the results in our patient indicate that chemotherapy is a reliable option for unresectable advanced MPUC.

In conclusion, we reported a case of MUC1-positive metastatic MPUC in which changes in CA 15-3 serum levels corresponded to the clinical course. Therefore, CA 15-3 serum level may be a potential biomarker for predicting aggressiveness and treatment response in patients with advanced MPUC.

\section{Acknowledgements}

The authors would like to thank Enago (www.enago.jp) for the English language review.

\section{Statement of Ethics}

Written informed consent was obtained from the patient for publication of this case report and the accompanying images.

\section{Conflict of Interest Statement}

The authors have no conflicts of interest to declare.

\section{Funding Sources}

The authors received no financial support for the research, authorship, or publication of this article.

\section{Karger'}




\section{Case Reports in Oncology}

Case Rep Oncol 2021;14:1019-1024

\begin{tabular}{l|l}
\hline DOI: $10.1159 / 000515781$ & @ 2021 The Author(s). Published by S. Karger AG, Basel
\end{tabular}
www.karger.com/cro

Takayama et al.: Cancer Antigen 15-3 as a Biomarker for Micropapillary Urothelial Carcinoma

\section{Author Contributions}

K.T. managed the case, redaction, and correction of the manuscript. S.N., Y.T., R.T., and T.H. assisted with redaction, correction, and reconstruction of the manuscript. All the authors read and approved the final manuscript.

\section{References}

1 Amin MB, Ro JY, el-Sharkawy T, Lee KM, Troncoso P, Silva EG, et al. Micropapillary variant of transitional cell carcinoma of the urinary bladder. Histologic pattern resembling ovarian papillary serous carcinoma. Am J Surg Pathol. 1994 Dec;18(12):1224-32.

2 Jin D, Jin K, Qiu S, Zhou X, Yuan Q, Yang L, et al. Prognostic values of the clinicopathological characteristics and survival outcomes in micropapillary urothelial carcinoma of the bladder: a SEER database analysis. Cancer Med. 2020 Jun;9:4897-906.

3 Pettinato G, Manivel CJ, Panico L, Sparano L, Petrella G. Invasive micropapillary carcinoma of the breast: clinicopathologic study of 62 cases of a poorly recognized variant with highly aggressive behavior. Am J Clin Pathol. 2004 Jun;121(6):857-66.

4 Duffy MJ. Biochemical markers in breast cancer: which ones are clinically useful? Clin Biochem. 2001 Jul; 34(5):347-52.

5 Colomer R, Ruibal A, Genolla J, Salvador L. Circulating CA 15-3 antigen levels in non-mammary malignancies. Br J Cancer. 1989 Feb;59(2):283-6.

6 Ahmad S, Lam TB, N’Dow J. Significance of MUC1 in bladder cancer. BJU Int. 2015 Jan;115(1):161-2.

7 Simms MS, Hughes OD, Limb M, Price MR, Bishop MC. MUC1 mucin as a tumour marker in bladder cancer. BJU Int. 1999 Aug;84:350-2.

8 Tsutsumida H, Nomoto M, Goto M, Kitajima S, Kubota I, Hirotsu Y, et al. A micropapillary pattern is predictive of a poor prognosis in lung adenocarcinoma, and reduced surfactant apoprotein A expression in the micropapillary pattern is an excellent indicator of a poor prognosis. Mod Pathol. 2007 Jun;20(6):638-47.

9 Sangoi AR, Higgins JP, Rouse RV, Schneider AG, McKenney JK. Immunohistochemical comparison of MUC1, CA125, and Her2Neu in invasive micropapillary carcinoma of the urinary tract and typical invasive urothelial carcinoma with retraction artifact. Mod Pathol. 2009 May;22(5):660-7.

10 Sanguedolce F, Russo D, Mancini V, Selvaggio O, Calo B, Carrieri G, et al. Prognostic and therapeutic role of HER2 expression in micropapillary carcinoma of the bladder. Mol Clin Oncol. 2019 Feb;10(2):205-13.

11 Guo CC, Dadhania V, Zhang L, Majewski T, Bondaruk J, Sykulski M, et al. Gene expression profile of the clinically aggressive micropapillary variant of bladder cancer. Eur Urol. 2016 Oct;70(49):611-20.

12 von Landenberg N, Speed JM, Trinh QD. Do micropapillary patients benefit from chemotherapy? BJU Int. 2017 May;119(5):656-8.

13 Warrick JI. Clinical significance of histologic variants of bladder cancer. J Natl Compr Canc Netw. 2017 Oct; 15(10):1268-74.

14 Fernández MI, Williams SB, Willis DL, Slack RS, Dickstein RJ, Parikh S, et al. Clinical risk stratification in patients with surgically resectable micropapillary bladder cancer. BJU Int. 2017 May;119(5):684-91.

15 Deuker M, Stolzenbach LF, Collà Ruvolo C, Nocera L, Mansour L, Tian Z, et al. Micropapillary versus urothelial carcinoma of the urinary bladder: stage at presentation and efficacy of chemotherapy across all stages-A SEER-based study. Eur Urol Focus. 2020 Sep 19;S2405-4569:30223-6. 